\title{
Zero-sum subsets of decomposable sets in Abelian groups
}

\author{
T. Banakh and A. Ravsky
}

Communicated by I. V. Protasov

\begin{abstract}
A subset $D$ of an abelian group is decomposable if $\varnothing \neq D \subset D+D$. In the paper we give partial answers to an open problem asking whether every finite decomposable subset $D$ of an abelian group contains a non-empty subset $Z \subset D$ with $\sum Z=0$. For every $n \in \mathbb{N}$ we present a decomposable subset $D$ of cardinality $|D|=n$ in the cyclic group of order $2^{n}-1$ such that $\sum D=0$, but $\sum T \neq 0$ for any proper non-empty subset $T \subset D$. On the other hand, we prove that every decomposable subset $D \subset \mathbb{R}$ of cardinality $|D| \leqslant 7$ contains a non-empty subset $T \subset D$ of cardinality $|Z| \leqslant \frac{1}{2}|D|$ with $\sum Z=0$. For every $n \in \mathbb{N}$ we present a subset $D \subset \mathbb{Z}$ of cardinality $|D|=2 n$ such that $\sum Z=0$ for some subset $Z \subset D$ of cardinality $|Z|=n$ and $\sum T \neq 0$ for any non-empty subset $T \subset D$ of cardinality $|T|<n=\frac{1}{2}|D|$. Also we prove that every finite decomposable subset $D$ of an Abelian group contains two non-empty subsets $A, B$ such that $\sum A+\sum B=0$.
\end{abstract}

\section{Introduction}

Our investigation is inspired by two problems posed in 2010 by Aryabhata on Mathematics StackExchange [1] and Gjergji Zaimi on MathOverflow [3]. Both problems remain open, despite the attacks of the members of these networks, who formulated and solved counterparts of the original problems and proposed different interpretations and reformulations of them, for instance, in terms of graphs and matrices. The problem posed

2010 MSC: 05E15.

Key words and phrases: decomposable set, abelian group, sum-set. 
by Gjergji Zaimi is a special case (for the additive group of real numbers) of our Problem 1, for which we obtain partial results in the present paper.

A subset $D$ of an Abelian group $G$ is called decomposable if $\varnothing \neq D \subset$ $D+D:=\{x+y: x, y \in D\}$. For a finite subset $F$ of an Abelian group put $\sum F=\sum_{x \in F} x$.

Problem 1. Let $D$ be a finite decomposable subset of an Abelian group $G$. Is $\sum Z=0$ for some non-empty set $Z \subset D$ ?

Observe that the answer to Problem 1 is affirmative if the group $G$ is Boolean (which means that $x+x=0$ for any $x \in G$ ). Indeed, take any element $a \in D$ and find elements $b, c \in D$ with $a=b+c$. If $0 \in\{a, b, c\}$, then $T=\{0\} \subset\{a, b, c\} \subset D$ is a subset with $\sum T=0$. If $0 \notin\{a, b, c\}$, then the elements $a, b, c$ are pairwise distinct and the set $T=\{a, b, c\}$ has $\sum T=a+b+c=2(b+c)=0$. Therefore, any decomposable set $D$ in a Boolean group contains a subset $T \subset D$ of cardinality $|T| \leqslant 3$ with $\sum T=0$.

This simple upper bound does not hold for decomposable sets in arbitrary groups.

Example 2. Let $n \geqslant 2$ and $G$ be a cyclic group of order $2^{n}-1$ with a generator $g$. The set $D=\left\{2^{k} g: 0 \leqslant k<n\right\}$ is decomposable and has $\sum D=0$ but $\sum T \neq \varnothing$ for any subset $T \subset D$ of cardinality $0<|T|<$ $|D|=n$.

Example 3. For every $n \in \mathbb{N}$ the subset

$$
D_{n}:=\left\{2^{k}: 0 \leqslant k<n\right\} \cup\left\{2^{k}-2^{n}+1: 0 \leqslant k<n\right\}
$$

of cardinality $\left|D_{n}\right|=2 n$ in the infinite cyclic group $\mathbb{Z}$ is decomposable and the subset

$$
Z=\left\{2^{k}: 1 \leqslant k<n\right\} \cup\left\{2-2^{n}\right\} \subset D_{n}
$$

of cardinality $|Z|=n$ has $\sum Z=0$. On the other hand, $\sum T \neq 0$ for every non-empty subset $T \subset D_{n}$ of cardinality $|T|<n$.

The properties of the set $D_{n}$ from Example 3 will be established in Section 1.

The above examples suggest to assign to every finite subset $D$ of an Abelian group $G$ the largest number $z(D) \leqslant|D|+1$ such that $\sum T \neq 0$ for any non-empty subset $T \subset D$ of cardinality $|T|<z(D)$. Therefore, $z(D)$ is equal to the smallest cardinality of a subset $Z \subset D$ with $\sum Z=0$ if such set $Z$ exists and $z(D)=|D|+1$ in the opposite case.

In terms of the number $z(D)$, Problem 1 can be reformulated as follows. 
Problem 4. Let $D$ be a finite decomposable subset of an Abelian group. Is $z(D) \leqslant|D|$ ?

The decomposable set $D_{n}$ from Example 2 has $z(D)=|D|=n$ and the decomposable set $D_{n} \subset \mathbb{Z}$ from Example 3 has $z\left(D_{n}\right)=n=\frac{1}{2}\left|D_{n}\right|$.

This suggests the following refinement of Problems 1 and 4 for the infinite cyclic group $\mathbb{Z}$.

Problem 5. Is $z(D) \leqslant \frac{1}{2}|D|$ for any finite decomposable subset $D \subset \mathbb{R}$ ?

A special case of this problem was posed by Aryabhata in [1].

Problem 6 (Aryabhata). Is $z(D) \leqslant 7$ for any decomposable subset $D \subset \mathbb{Z}$ of cardinality $|D|=15$ ?

In Section 2 we shall provide an affirmative answer to Problem 5 for decomposable subsets $D \subset \mathbb{Z}$ of cardinality $|D| \leqslant 7$.

Proposition 7. Any decomposable subset $D \subset \mathbb{R}$ of cardinality $|D| \leqslant 7$ has $z(D) \leqslant \frac{1}{2}|D|$.

We also observe that for $n \in\{2,3\}$ the decomposable set $D_{n}=$ $\left\{2^{k}, 2^{k}-2^{n}+1: 0 \leqslant k<n\right\}$ form Example 3 is unique in the following sense.

Proposition 8. Every decomposable set $D \subset \mathbb{R}$ with $n=z(D)=\frac{1}{2}|D| \in$ $\{2,3\}$ is equal to $D_{n} \cdot r$ for some real number $r$.

Problem 9. Is every finite decomposable set $D \subset \mathbb{R}$ with $z(D)=\frac{1}{2}|D|=$ $n \geqslant 2$ equal to $D_{n} \cdot r$ for some real number $r$ ?

The following proposition shows that the problems on finite decomposable sets in torsion-free Abelian groups can be reduced to the case of the infinite cyclic group.

Proposition 10. For any finite decomposable set $D$ in a torsion-free Abelian group $G$ there exists a decomposable set $D^{\prime} \subset \mathbb{Z}$ such that $\left|D^{\prime}\right|=$ $|D|$ and $z\left(D^{\prime}\right)=z(D)$.

Proof. We lose no generality assuming that $G$ is finitely-generated and hence is isomorphic to $\mathbb{Z}^{n}$ for some $n \in \mathbb{N}$. Let $e_{1}, \ldots, e_{n}$ be the standard generators of the group $\mathbb{Z}^{n}$. Find $m \in \mathbb{N}$ such that $\left\{\sum F: F \subset D\right\} \subset$ $\left\{\sum_{i=1}^{n} x_{i} e_{i}:\left(x_{i}\right)_{i=1}^{n} \in[-m, m]^{n}\right\}=[-m, m]^{n}$. Consider the homomorphism $h: \mathbb{Z}^{n} \rightarrow \mathbb{Z}$ such that $h\left(e_{i}\right)=(2 m+1)^{i}$ for all $1 \leqslant i \leqslant n$. It is easy to see that the restriction $h \uparrow[-m, m]^{n}$ is injective. Consequently, a subset $F \subset D$ has $\sum F=0$ if and only if $\sum h(F)=0$. This implies that for the set $D^{\prime}=h(D)$ we have the equalities $\left|D^{\prime}\right|=|D|$ and $z\left(D^{\prime}\right)=z(D)$. 
Our final result provides an affirmative answer to a weak version of Problem 1. The following theorem will be proved in Section 3.

Theorem 11. For any finite decomposable subset $D$ of an Abelian group there are two non-empty sets $A, B \subset D$ such that $\sum A+\sum B=0$.

Corollary 12. For any finite decomposable subset $D$ of an Abelian group, there exists a non-empty subset $T \subset D$ and a function $f: T \rightarrow\{1,2\}$ such that $\sum_{x \in T} f(x) \cdot x=0$.

A decomposable subset $D$ of an Abelian group is called minimal decomposable if no proper subset of $D$ is decomposable. It is clear that every finite decomposable set contains a minimal decomposable set.

Corollary 12 can be compared with the following result that was essentially proved by Hsien-Chih Chang [2] in his answer to the problem of Zaimi [3].

Proposition 13. For every finite minimal decomposable subset $D$ of an Abelian group there exists a function $f: D \rightarrow \omega$ such that $\sum_{x \in D} f(x)=$ $|D|$ and $\sum_{x \in D} f(x) \cdot x=0$.

Proof. By the decomposability of $D$, there exist functions $\alpha, \beta: D \rightarrow D$ such that $x=\alpha(x)+\beta(x)$ for every $x \in X$. For every $x \in D$ let $g(x)=$ $\left|\alpha^{-1}(x)\right|+\left|\beta^{-1}(x)\right|$ and observe that $\sum_{x \in D} g(x)=|D|+|D|$. The minimal decomposability of $D$ ensures that $D=\alpha(D) \cup \beta(D)$ and hence $f(x):=$ $g(x)-1 \geqslant 0$ for every $x \in D$. Then $\sum_{x \in D} f(x)=(|D|+|D|)-|D|=|D|$. It follows that $\sum D=\sum_{x \in D}(\alpha(x)+\beta(x))=\sum_{y \in D} g(y) \cdot y$ and hence $\sum_{x \in D} f(x) \cdot x=\sum_{x \in D}(g(x)-1) \cdot x=0$.

\section{Properties of the decomposable set in Example 3}

Given a natural number $n$ consider the subsets $A=\left\{1,2,4, \ldots, 2^{n-1}\right\}$ and

$$
D_{n}:=A \cup\left(A-\left(2^{n}-1\right)\right)
$$

in the group $\mathbb{Z}$ of integers. Then $\left|D_{n}\right|=2 n$.

The set $D_{n}$ is decomposable, because, clearly, each element of the set $A \backslash\{1\}$ is decomposable, $1=2^{n-1}+\left(2^{n-1}-\left(2^{n}-1\right)\right), 2^{k}-\left(2^{n}-1\right)=$ $2^{k-1}+\left(2^{k-1}-\left(2^{n}-1\right)\right)$ for every positive $k<n$, and $1-\left(2^{n}-1\right)=$ $\left(2^{n-1}-\left(2^{n}-1\right)\right)+\left(2^{n-1}-\left(2^{n}-1\right)\right)$.

It is clear that the set $Z=\left\{2^{k}: 1 \leqslant k<n\right\} \cup\left\{2^{n}-2\right\}$ has cardinality $|Z|=n=\frac{1}{2}\left|D_{n}\right|$ and $\sum Z=0$. 
Next, we prove that every subset $T \subset D_{n}$ of cardinality $|T|<n$ has $\sum T \neq 0$. Assuming that $\sum T=0$, we conclude that $n \geqslant 3$ and $T$ contains at most $n-2$ positive elements. Since all of them are distinct elements of $A$, their sum is at most $2^{n}-4$. On the other hand, the largest negative element of the set $A-\left(2^{n}-1\right)$ is $-2^{n-1}+1=-\left(2^{n}-2\right) / 2$. Thus if $T$ contains at least two negative elements then $\sum T<0$. If $T$ contains exactly one negative element $2^{k}-2^{n}+1$ then $\sum T=0$ implies that we have a representation of $2^{n}-1$ as a sum of at most $n-1$ powers of 2 with at most one power used twice. This representation collapses to a sum of at most $n-1$ distinct powers of 2 . If the representation contains a power $2^{l}$ with $l \geqslant n$ then it is bigger than $2^{n}-1$. Otherwise the sum is at most $2^{1}+2^{2}+\cdots+2^{n-1}=2^{n}-2<2^{k}-1$. Thus $z\left(D_{n}\right) \geqslant n$.

\section{Proof of Propositions 7 and 8}

We divide the proof of Propositions 7 and 8 into five lemmas. In fact, Proposition 8 follows from Lemmas 15 and 18 proved below.

Lemma 14. Every decomposable subset $D \subset \mathbb{R}$ of cardinality $|D| \leqslant 3$ contains zero and hence has $z(D)=1 \leqslant \frac{1}{2}|D|$.

Proof. To derive a contradiction, assume that $0 \notin D$. Replacing $D$ by $-D$, if necessary, we can assume that $D$ contains a unique positive element $p$. Since $p=a+b$ for some elements $a, b \in D$, one of the numbers $a$ or $b$ is positive, so it equals $p$ and the other summand is zero.

The following lemma was proved by Ingdas [3]. We present a short proof for convenience of the reader.

Lemma 15 (Ingdas). Any decomposable subset $D \subset \mathbb{R}$ with $|D|=4$ and $z(D) \geqslant 2$ is equal to the set $\{-2,-1,1,2\} \cdot r=D_{2} \cdot r$ for some positive real number $r$.

Proof. Since $z(D) \geqslant 2$, the set $D$ does not contain zero. Then $D$ should contains at least two positive numbers and at least two negative numbers (otherwise $D$ will be not decomposable). Since $|D|=4$, the set $D$ contains exactly two positive and two negatice numbers. Let $c$ be the largest positive element of $D$. Since $D$ is decomposable, $c=a+b$ for some $a, b \in D$. Since $D$ does not contain zero, the maximality of $c$ ensures that the elements $a, b$ are strictly positive and hence coincide with the unique positive element $r$ of the set $D \backslash\{c\}$. Therefore, $c=a+b=2 r$. By the same reason, the subset $\{x \in D: x<0\}$ of negative numbers is 
equal to $\{2 n, n\}$ for some negative real number $n$. Write the element $r$ as $r=x+y$ for some $x, y \in D$ with $x \leqslant y$. Taking into account that $D$ contains no zero and $r, 2 r$ are unique positive elements of $D$, we conclude that $y=2 r$ and then $x=r-y=r-2 r=-r \in\{2 n, n\}$. If $-r=2 n$, then $D=\left\{-r,-\frac{1}{2} r, r, 2 r\right\}$ is not decomposable as $-\frac{1}{2} r \notin D+D$. So, $r=-n$ and hence $D=\{-2 r,-r, r, 2 r\}=\{-2,-1,1,2\} \cdot r$.

Lemma 16. Any decomposable subset $D \subset \mathbb{R}$ of cardinality $|D| \in\{4,5\}$ contains a subset $T \subset D$ of cardinality $|Z| \in\{1,2\}$ with $\sum Z=0$. Consequently, $z(D) \leqslant 2 \leqslant \frac{1}{2}|D|$.

Proof. If $0 \in D$, then $Z=\{0\}$ has $\sum Z=0$ and witnesses that $z(D)=$ $0 \leqslant \frac{1}{2}|D|$.

So, assume that $0 \notin D$. Replacing $D$ by $-D$ we can assume that $D$ has at most two positive elements. Let $c$ be the largest positive element of $D$. Since $D$ is decomposable, $c=a+b$ for some $a, b \in D$. Since $D$ does not contain zero, the maximality of $c$ ensures that the elements $a, b$ are strictly positive and hence coincide with the unique positive element of the set $D \backslash\{c\}$. Therefore, $c=a+b=2 a$. Write the element $a$ as $a=x+y$ for some $x, y \in D$ with $x \leqslant y$. Taking into account that $D$ contains no zero and $a, c$ are unique positive elements of $D$, we conclude that $y=c$ and then $x=a-y=a-c=a-2 a=-a$. Then the set $Z=\{-a, a\}$ has $\sum Z=0$ and witnesses that $z(D) \leqslant 2 \leqslant \frac{1}{2}|D|$.

Remark 17. It can be shown that each decomposable subset $D \subset \mathbb{R}$ of cardinality $|D|=5$ with $0 \notin D$ is equal to one of the sets

$$
\{-3,-2,-1,1,2\} \cdot r, \quad\{-4,-2,-1,1,2\} \cdot r, \quad\{-3,-2,-1,2,4\} \cdot r
$$

for some non-zero number $r$.

Lemma 18. Any decomposable subset $D \subset \mathbb{R}$ of cardinality $|D|=6$ with $z(D) \geqslant 3$ coincides with $\{1,2,4,-3,-5,-6\} \cdot r$ for some real number $r$ and hence has $z(D)=3=\frac{1}{2}|D|$.

Proof. Let $D$ be a decomposable set consisting of six real numbers. If $0 \in D$ then $z(D)=1$. If $D$ contains exactly one (resp. two) positive (or negative) elements then similarly to the case $n \leqslant 3$ (resp. $4 \leqslant n \leqslant 5$ ) we can show that $z(D)=1$ (resp. $z(D) \leqslant 2$ ).

So it remains to consider the case when $D$ consists of three positive and three negative numbers. Let $p_{\max }$ (resp. $n_{\min }$ ) be the largest positive (resp. the smallest negative) element of $D$. Write $p_{\max }$ as $p_{\max }=p+p^{\prime}$ 
for some numbers $p, p^{\prime} \in D$ with $p \leqslant p^{\prime}$. By the maximality of $p_{\max }$, both numbers $p$ and $p^{\prime}$ are positive. Assume that $p<p^{\prime}$ and write $p=\bar{p}+a$, $p^{\prime}=\bar{p}^{\prime}+a^{\prime}$ for some elements of $D$ with positive $\bar{p}$ and $\bar{p}^{\prime}$. If $\bar{p}=p_{\max }$ (resp. $\bar{p}^{\prime}=p_{\max }$ ) then $p^{\prime}+a=0$ (resp. $p+a^{\prime}=0$ ), so $z(D)=2$. Therefore, we can assume that $\bar{p} \neq p_{\max } \neq \bar{p}^{\prime}$. In this case $\bar{p}=p^{\prime}$ and $\bar{p}^{\prime}=p$. Consequently, $a=p-\bar{p}=p-p^{\prime}=\bar{p}^{\prime}-p^{\prime}=-a^{\prime}$ and $Z=\left\{a, a^{\prime}\right\}$ is a set with $\sum Z=0$, witnessing that $z(D) \leqslant 2$.

Thus it remains to consider the case when $p_{\max }=2 p^{\prime}$ for some $p^{\prime} \in D$ and, by the symmetry, $n_{\min }=2 n^{\prime}$ for some $n^{\prime} \in D$. Let $p^{\prime}, 2 p^{\prime}=p_{\max }, p^{\prime \prime}$ be positive elements of the set $D$ and $n^{\prime}, 2 n^{\prime}=n_{\min }, n^{\prime \prime}$ be its negative elements. Then $n^{\prime}$ is a sum of two elements of $D$ at least one of which is negative. If $n^{\prime}=2 n^{\prime}+x$ for some $x \in D$ then $n^{\prime}+x=0$ and $z(D)=2$. Thus $n^{\prime}=n^{\prime \prime}+a$ for some $a \in D$. If $n^{\prime \prime}=n^{\prime}+b$ for some $b \in D$ then $a+b=0$ and $z(D)=2$. Thus $n^{\prime \prime}=2 n^{\prime}+\bar{p}$ for some positive $\bar{p} \in D$. Similarly, $p^{\prime}=p^{\prime \prime}+a^{\prime}$ for some $a^{\prime} \in D$ and $p^{\prime \prime}=2 p^{\prime}+\bar{n}$ for some negative $\bar{n} \in D$. If $a>0$ and $a^{\prime}<0$ then $\max \left\{\bar{n}, a^{\prime}\right\} \leqslant n^{\prime}$ and $p^{\prime} \leqslant \min \{\bar{p}, a\}$. So

$$
0=n^{\prime}+a+\bar{p} \geqslant n^{\prime}+p^{\prime}+p^{\prime}>p^{\prime}+n^{\prime}>p^{\prime}+n^{\prime}+n^{\prime} \geqslant p^{\prime}+a^{\prime}+\bar{n}=0,
$$

a contradiction. Thus $a<0$ or $a^{\prime}>0$. If $a<0$, then since $n^{\prime}=n^{\prime \prime}+a$ we have $n^{\prime}=2 n^{\prime \prime}$. Similarly, if $a^{\prime}>0$, then $p^{\prime}=2 p^{\prime \prime}$. Reverting the signs of elements of $D$, if needed, we can suppose that $p^{\prime}=2 p^{\prime \prime}$. Then $\left\{p^{\prime \prime}, 2 p^{\prime \prime}, 4 p^{\prime \prime}\right\} \in D$, so $\bar{n}=-3 p^{\prime \prime}$ and $n^{\prime \prime}=\bar{p}+2 n^{\prime}$. The following cases are possible:

1. $n^{\prime}=\bar{n}=-3 p^{\prime \prime}$, so $2 n^{\prime}=-6 p^{\prime \prime}$.

1.1. If $\bar{p}=p^{\prime \prime}$ then $n^{\prime \prime}=-5 p^{\prime \prime}$ so the proposition claim holds.

1.2. If $\bar{p}=2 p^{\prime \prime}$ then $n^{\prime \prime}=-4 p^{\prime \prime}$, so $n^{\prime \prime}+4 p^{\prime \prime}=0$ and $z(D)=2$.

1.3. If $\bar{p}=4 p^{\prime \prime}$ then $n^{\prime \prime}=-2 p^{\prime \prime}$, so $n^{\prime \prime}+2 p^{\prime \prime}=0$ and $z(D)=2$.

2. $2 n^{\prime}=\bar{n}=-3 p^{\prime \prime}$, so $n^{\prime}=-1.5 p^{\prime \prime}$.

2.1. If $\bar{p}=p^{\prime \prime}$ then $n^{\prime \prime}=-2 p^{\prime \prime}$, so $n^{\prime \prime}+2 p^{\prime \prime}=0$ and $z(D)=2$.

2.2. If $\bar{p}=2 p^{\prime \prime}$ then $n^{\prime \prime}=-p^{\prime \prime}$, so $n^{\prime \prime}+p^{\prime \prime}=0$ and $z(D)=2$.

2.3. If $\bar{p}=4 p^{\prime \prime}$ then $n^{\prime \prime}=p^{\prime \prime}>0$, a contradiction.

3. $n^{\prime \prime}=\bar{n}=-3 p^{\prime \prime}$. Then $\bar{p}+2 n^{\prime}+3 p^{\prime \prime}=0$.

3.1. If $\bar{p}=p^{\prime \prime}$ then $n^{\prime}=-2 p^{\prime \prime}$, so $n^{\prime}+2 p^{\prime \prime}=0$ and $z(D)=2$.

3.2. If $\bar{p}=2 p^{\prime \prime}$ then $n^{\prime}=-2.5 p^{\prime \prime}$, so $2 n^{\prime \prime}=-5 p^{\prime \prime}$ and $n^{\prime} \notin D+D$, a contradiction.

3.3. If $\bar{p}=4 p^{\prime \prime}$ then $n^{\prime}=-3.5 p^{\prime \prime}$, so $2 n^{\prime \prime}=-7 p^{\prime \prime}$ and $n^{\prime} \notin D+D$, a contradiction.

Lemma 19. Every decomposable subset $D \subset \mathbb{R}$ of cardinality $|D|=7$ has $z(D) \leqslant 3 \leqslant \frac{1}{2}|D|$. 
Proof. Let $D$ be a decomposable set consisting of seven real numbers. If $0 \in D$ then $z(D)=1$. If $D$ contains exactly one (resp. two) positive (or negative) elements then similarly to the case $n \leqslant 3$ (resp. $4 \leqslant n \leqslant 5$ ) we can show that $z(D)=1$ (resp. $z(D) \leqslant 2$ ). So, reverting the signs of elements of $D$, if needed, it remains to consider the case when $D$ consists of four positive and three negative numbers. Let $p_{\max }$ (resp. $n_{\min }$ ) be the largest positive (resp. the smallest negative) element of $D$. Similarly to the proof of Lemma 18 we can show that $n_{\text {min }}=2 n^{\prime}$ for some $n^{\prime} \in D$.

Let $n^{\prime}, 2 n^{\prime}=n_{\min }, n^{\prime \prime}$ be negative elements of the set $D$. Similarly to the proof of Lemma 18 we can show that $n^{\prime}=n^{\prime \prime}+a$ for some $a \in D$ and $n^{\prime \prime}=2 n^{\prime}+\bar{p}$ for some positive $\bar{p} \in D$. Then $n^{\prime}+a+\bar{p}=0$. If these numbers are distinct then $z(D) \leqslant 3$. So we assume the converse. Since $n^{\prime}=n^{\prime \prime}+a, a \neq n^{\prime}$, so $a=\bar{p}=n^{\prime}-n^{\prime \prime}=n^{\prime \prime}-2 n^{\prime}$ and $3 n^{\prime}=2 n^{\prime \prime}$. Divide all elements of the set $D$ by $\frac{1}{2}\left|n^{\prime}\right|$. Then it will have elements $-2,-3,-4$, and 1 .

Depending on the representation of 1 as a sum of elements of $D$, the following cases are possible:

$1.1=3-2$, and $3 \in D$. Since $-3 \in D$, we have $z(D) \leqslant 2$.

2 . $1=4-3$, and $4 \in D$. Since $-4 \in D$, we have $z(D) \leqslant 2$.

3. $1=5-4$, and $5 \in D$. Since $-2,-3 \in D$, we have $z(D) \leqslant 3$.

4. 1 is a sum of distinct positive elements of $D$. Then $p_{\max }<2$ so a sum of each positive and negative elements of $D$ is negative. Then the smallest positive element of $D$ does not belong to $D+D$, a contradiction.

$5.1=0.5+0.5$ and $0.5 \in D$. If the smallest positive element $p_{\min }$ of $D$ is less than 0.5 then $p_{\max } \leqslant 1+1=2$. Then a sum of each positive and negative elements of $D$ is non-positive and $p_{\min } \notin D+D$, a contradiction. Thus $p_{\min }=0.5$ and one of numbers $2.5,3.5$, and 4.5 belongs to $D$. But $2.5+0.5+(-3)=0$ and $3.5+0.5+(-4)=0$, so either $z(D) \leqslant 3$ or 4.5 belongs to $D$. We assume the last case. Then $D^{\prime}=\{-4,-3,-2,0.5,1,4.5\} \subset D$. Let $p$ be a unique element of $D \backslash D^{\prime}$. Since $D^{\prime}+D^{\prime} \not \ngtr 4.5,4.5=p+s$ for some $s \in D$. Since $p \geqslant 4.5 / 2=2.25$, $p \notin D^{\prime}+D^{\prime}$ and hence $p=4.5+s^{\prime}$ for some $s^{\prime} \in D$. Then $s+s^{\prime}=0$ and so $z(D) \leqslant 2$.

\section{Proof of Theorem 11}

First we introduce some notation. For a function $f: X \rightarrow Y$ and subset $A \subset X$ put $f[A]=\{f(a): a \in A\}$. 
By a tree we understand any non-empty finite partially ordered set $(T, \leqslant)$ such that for every $x \in T$ the set $\downarrow x=\{t \in T: t \leqslant x\}$ is linearly ordered.

Let $T$ be a tree. By min $T$ we denote the smallest element of $T$ and by $\max T$ the set of all maximal elements of $T$. A branch in a tree is a maximal linearly ordered subset $B \subset T$, which can be identified with the largest element of $B$.

For an element $x$ of a tree $T$ let $\uparrow x:=\{y \in T: x \leqslant y\}$ and $\operatorname{succ}_{T}(x):=$ $\min (\uparrow x \backslash\{x\})$ be the set of immediate successors of $x$ in the tree $T$. For any $x \in \max T$ we have $\operatorname{succ}_{T}(x)=\varnothing$. For any element $x \neq \min T$ let $\operatorname{prec}_{T}(x)$ be the unique element $y \in T$ such that $x \in \operatorname{succ}_{T}(y)$.

A tree $T$ is called binary if for each $x \in T \backslash \max T$ the $\operatorname{set} \operatorname{succ}_{T}(x)$ has cardinality 2.

For a branch $B$ in a binary tree, let $\perp_{B}: B \backslash \max B \rightarrow T$ be the function assigning to each element $x \in B \backslash \max B$ the unique element of the set $\operatorname{succ}_{T}(x) \backslash B$.

A function $f: \max T \rightarrow T$ is called regressive if $f(x)<x$ for each $x \in \max T$.

Lemma 20. For any regressive function $f: \max T \rightarrow T$ on a binary tree $T$ there are distinct elements $x, y \in \max T$ such that $f(x)=f(y)=$ $\max (\downarrow x \cap \downarrow y)$.

Proof. The proof if by induction on the height $\hbar(T):=\max \{|\downarrow x|: x \in T\}$ of the binary tree $T$. If $\hbar(T)=1$, then no regressive function $f: \max T \rightarrow$ $T$ exists, so the statement of the lemma holds.

Assume that the lemma has been proved for all binary trees of height $<n$. Take a binary tree $T$ of height $n>1$. Let $\min T$ be the root of $T$ and $x_{1}, x_{2}$ be two immediate successors of $\min T$ in $T$. Then $T_{1}:=\uparrow x_{1} \backslash\{\min T\}$ and $T_{2}:=\uparrow x_{2} \backslash\{\min T\}$ are trees of height $<n$. Two cases are possible.

1. $f\left(\max T_{i}\right) \subset T_{i}$ for some $i \in\{1,2\}$. In this case we can apply the inductive assumption and find two elements $x, y \in \max T_{i}$ such that $f(x)=f(y)=\max (\downarrow x \cap \downarrow y)$.

2. For every $i \in\{1,2\}$ there exists an element $t_{i} \in \max T_{i}$ such that $f\left(t_{i}\right)=\min T$. Then $x=t_{1}$ and $y=t_{2}$ are two elements with $f(x)=f(y)=\max (\downarrow x \cap \downarrow y)$.

Now we can present the proof of Theorem 11. Given a finite decomposable subset $D$ of an Abelian group, we should find two non-empty subsets $A, B \subset D$ with $\sum A+\sum B=0$. 
Let $D$ be a finite subset of an abelian group with $D \subset D+D$. By a binary $D$-tree we understand a pair $(T, d)$ consisting of a binary tree $T$ and a function $d: T \rightarrow D$ such that each non-maximal element $x \in T$ we have $d(x)=d(y)+d(z)$, where $\{y, z\}=\operatorname{succ}_{T}(x)$. A binary $D$-tree is called $\perp$-injective if for each branch $L \subset T$ the restriction $d\left\lceil\perp_{L}[L \backslash \max L]\right.$ is injective. This implies that $\left|\perp_{L}[L \backslash \max L]\right| \leqslant|D|$ and hence $|L| \leqslant|D|+1$. Consequently, each $\perp$-injective binary $D$-tree is finite, so we can choose a maximal $\perp$-injective binary $D$-tree $T$. Since $D \subset D+D$, the tree $T$ is a subtree of a binary $D$-tree $\tilde{T}=T \cup \max \tilde{T}$ such that $T \cap \max \tilde{T}=\varnothing$. The maximality of the tree $T$ ensures that for any $x \in \max T$, there exists an element $x^{\prime} \in \operatorname{succ}_{\tilde{T}}(x)$ such that $d\left(x^{\prime}\right) \in d\left[\perp_{\downarrow x}[\downarrow x \backslash\{x\}]\right.$. Let $M_{2}=\left\{x \in \max T: d\left[\operatorname{succ}_{\tilde{T}}(x)\right] \subset d\left[\perp_{\downarrow x}[\downarrow x \backslash\{x\}]\right\}\right.$ and $M_{1}=\max T \backslash M_{2}$.

For every $x \in M_{1}$ let $x_{1}$ be the unique immediate successor of $x$ such that

$$
d\left(x_{1}\right) \in d\left[\perp_{\downarrow x}[\downarrow x \backslash\{x\}]\right]
$$

and $g(x) \in \downarrow x$ be a (unique) point such that $d\left(x_{1}\right)=d\left(\perp_{\downarrow x}(g(x))\right)$. Let $f(x):=g(x)$.

For every $x \in M_{2}$ and every point $x^{\prime} \in \operatorname{succ}_{\tilde{T}}(x)$ there is a point $g\left(x^{\prime}\right) \in \downarrow x$ such that $d\left(x^{\prime}\right)=d\left(\perp_{\downarrow x}\left(g\left(x^{\prime}\right)\right)\right)$. Let $f(x):=\max \left\{g\left(x^{\prime}\right): x^{\prime} \in\right.$ $\left.\operatorname{succ}_{\tilde{T}}(x)\right\}$.

Now observe that we have defined a regressive function $f: \max T \rightarrow T$. By Lemma 20, there are two maximal elements $x, y \in \max T$ such that $f(x)=f(y)=\max (\downarrow x \cap \downarrow y)$.

By the definition of $f(x)$ the set $\operatorname{succ}_{\tilde{T}}(x)$ contains a point $x_{1}$ such that $f(x)=g\left(x_{1}\right)$. Let $x_{2}$ be the unique point of $\operatorname{succ}_{\tilde{T}}(x) \backslash\left\{x_{1}\right\}$. The definition of the function $f$ guarantees that $d\left(x_{2}\right) \notin\left\{d\left(\perp_{\downarrow x}(t)\right): f(x)<t<x\right\}$. By analogy the $\operatorname{set}^{\operatorname{succ}_{\tilde{T}}}(y)$ can be written as $\left\{y_{1}, y_{2}\right\}$ such that and $f(y)=g\left(y_{1}\right)$ and $d\left(y_{2}\right) \notin\left\{d\left(\perp_{\downarrow y}(t)\right): f(y)<t<y\right\}$.

Let $g^{\prime}\left(x_{1}\right)=\perp_{\downarrow x}\left(g\left(x_{1}\right)\right)=\perp_{\downarrow x}(f(x))$ and $g^{\prime}\left(y_{1}\right)=\perp_{\downarrow y}\left(g\left(y_{1}\right)\right)=$ $\perp_{\downarrow y}(f(y))$. Observe that $\left\{g^{\prime}\left(x_{1}\right), g^{\prime}\left(y_{1}\right)\right\}=\operatorname{succ}_{T}(f(x)), g^{\prime}\left(x_{1}\right) \in \downarrow y$ and $g^{\prime}\left(y_{1}\right) \in \downarrow x$.

Let

$$
A_{T}=\left\{\perp_{\downarrow x}(t): f(x)<t<x\right\} \text { and } B_{T}:=\left\{\perp_{\downarrow y}(t): f(y)<t<y\right\} .
$$

It follows from the definition of $f(x)=f(y)$ that $d\left(x_{2}\right) \notin d\left[A_{T}\right]$ and $d\left(y_{2}\right) \notin d\left[B_{T}\right]$.

By induction it can be shown that

$$
d\left(y_{1}\right)=d\left(g^{\prime}\left(y_{1}\right)\right)=d\left(x_{1}\right)+d\left(x_{2}\right)+\sum_{t \in A_{T}} d(t)
$$


and

$$
d\left(x_{1}\right)=d\left(g^{\prime}\left(x_{1}\right)\right)=d\left(y_{1}\right)+d\left(y_{2}\right)+\sum_{t \in B_{T}} d(t) .
$$

Then

$$
d\left(y_{1}\right)-d\left(x_{1}\right)=\sum_{t \in A_{T} \cup\left\{x_{2}\right\}} d(t)=-\sum_{t \in B_{T} \cup\left\{y_{2}\right\}} d(t)
$$

and finally $\sum A=-\sum B$ for the sets $A=d\left[A_{T}\right] \cup\left\{d\left(x_{2}\right)\right\}$ and $B=$ $d\left[B_{T}\right] \cup\left\{d\left(y_{2}\right)\right\}$.

\section{References}

[1] Aryabhata et al., A zero sum subset of a sum-full set, Mathematics StackExchange, math. stackexchange.com/q/2418.

[2] Hsieh-Chih Chang, A partial answer to a problem of Zaimi, MathOverflow, mathoverflow.net/a/16871.

[3] Gjergji Zaimi et al., Existence of a zero-sum subset, MathOverflow, mathoverflow. net/q/16857.

\section{CONTACT INFORMATION}

Taras Banakh

Alex Ravsky
Faculty of Mechanics and Mathematics, Ivan

Franko National University, Lviv, Universytet'ska 1, 79602, Ukraine, and Jan Kochanowski University in Kielce, Poland E-Mail(s): t.o.banakh@gmail.com Web-page(s): prima.Inu.edu.ua/faculty/ mechmat/Departments/ Topology/bancv.html

Department of Analysis, Geometry and Topology, Pidstryhach Institute for Applied Problems of Mechanics and Mathematics National Academy of Sciences of Ukraine, Naukova 3-b, Lviv, 79060, Ukraine E-Mail(s): alexander.ravsky@uni-wuerzburg.de

Received by the editors: 18.11.2019

and in final form 21.03.2020. 